Revue d'histoire de l'Amérique française

ZRS REVUE D.HISTOIRE DE L'AMÉRIQUE FRANÇAISE

\title{
Itinéraire spirituel de Marguerite Bourgeoys (suite)
}

\section{Yvon Charron}

Volume 2, numéro 3, décembre 1948

URI : https://id.erudit.org/iderudit/801475ar

DOI : https://doi.org/10.7202/801475ar

Aller au sommaire du numéro

Éditeur(s)

Institut d'histoire de l'Amérique française

ISSN

0035-2357 (imprimé)

1492-1383 (numérique)

Découvrir la revue

Citer cet article

Charron, Y. (1948). Itinéraire spirituel de Marguerite Bourgeoys (suite). Revue d'histoire de l'Amérique française, 2(3), 351-374.

https://doi.org/10.7202/801475ar d'utilisation que vous pouvez consulter en ligne.

https://apropos.erudit.org/fr/usagers/politique-dutilisation/ 


\title{
ITINÉRAIRE SPIRITUEL DE MARGUERITE BOURGEOYS*
}

\author{
( suite et fin)
}

II

SECONDE CONVERSION

(1640-70)

Glandelet, en parlant du charisme dont Marguerite fut la bénéficiaire ce premier dimanche d'octobre 1640 use du mot: conversion, mais non, toutefois, sans apporter un correctif qui a pour résultat de donner sa vraie mesure à l'événement qui se produisit alors:

Elle se convertit entièrement à Dieu, si toutefois on doit appeler proprement $\mathrm{du}$ nom de conversion le passage d'une vie innocente à une vie pleine de faveurs. ${ }^{18}$

A vrai dire cet événement constitue une certaine volte-face dans la vie spirituelle de Marguerite qui à compter de ce jour tourne résolument le dos à la politique de compromis et de tergiversations qui avait été un peu la sienne dans le service de Dieu. Elle commence de se plier, sans lésiner, aux dures exigences de l'idéal de sainteté qui la sollicite depuis longtemps. De commençante - ou mieux d'hésitante elle devient progressante et franchit ce que l'on est convenu d'appeler dans l'école le seuil de la vie illuminative. Par là même s'inaugure pour elle une période de purifications nouvelles, actives et passives, période longue de trente années (1640-1670) qui, croyons-nous, la mettra vers l'année 1670 aux portes de la vie unitive. C'est en effet vers cette époque qu'elle entrera dans la Nuit de l'esprit après avoir passé par la nuit des sens.

*Voir Revue d'Histoire de l'A mérique française (septembre 1948) p. 230-37.

18. VSMB., 4. Marguerite Bourgeoys ne parle, et plus justement, que de se a donner à Dieu ". EA., 127, 141. 
$1640-1652$

Donc le dimanche 7 octobre 1640, il y avait procession au Couvent des Jacobins de Troyes, en l'honneur de la Vierge du Rosaire. L'affluence du peuple fut telle que le cortège ne put demeurer à l'intérieur du cloître et la procession prit la rue pour passer devant l'abbaye de Notre-Dame-aux-Nonnains. C'était là que la grâce de Dieu attendait Marguerite. L'abbaye possédait une entrée principale dont le portail s'appelait à raison de sa splendeur: le Beau Portail. Une statue en pierre de la Vierge Marie y prédominait:

En jetant la veue pour la regarder je la trouvay tres belle et en mesme temps je me trouva si touchee et si changee que je ne me connoissois plus et retournant a la maison cela paroissoit à tous. $^{19}$

Tout un aspect nous échappe du phénomène qui se produsit alors. Y eut-il apparition véritable avec ou sans transformation miraculeuse de la statue de la Vierge? Nous ne savons. L'extase fut-elle le fait de Marguerite ? Peut-être; et même plus probablement il en fut ainsi. Mais enfin rien ne permet, selon nous, de l'affirmer avec certitude. ${ }^{20}$ Le laconisme des Écrits autographes n'autorise que des probabilités. Mais, à vrai dire, l'essentiel n'est pas là. Ce qui n'est pas douteux, c'est qu'en un instant l'âme de la jeune fille se trouva être sous l'emprise d'une grâce éminente qui la souleva toute. Sous l'influence de l'un ou l'autre des dons du Saint-Esprit - celle du don de sagesse, semble-t-il - elle entrevit la beauté divine transfigurant dans son âme à elle les traits de la Vierge Marie. Dans le même faisceau de lumières infuses, elle mesura aussi la médiocrité relative de la vie chrétienne qui avait été la sienne jusque-là et porta jugement avec la sévérité de ceux qui pour de bon sont épris de vie divine.

C'en était fait: Marguerite Bourgeoys était gagnée à Dieu et de façon décisive. Sans doute elle ne faisait que débuter dans la série des purifications passives auxquelles Dieu soumet les âmes qui veulent pousser loin dans la voie de la sainteté; mais quand même elle était en marche, et le revirement qui venait de s'opérer en elle, allait

19. EA., 126-127, 27; - aussi Glandelet, VSMB., 4-5.

20. Nous observons que Dom Jamet est plus catégorique; selon lui « unstant il y eut extase , Marguerite Bourgeoys, 1:30. 
comporter des conséquences non équivoques. Est-il besoin de remarquer ici que les théologiens caractérisent l'entrée des spirituels dans la vie illuminative par un revirement de cette sorte?

Chez les progressants, avec le deuxième degré de la charité, apparaissent les vertus solides et non plus seulement les vertus initiales, en particulier la douceur et la patience, une humilité vraie, qui porte à la bienveillance sur le prochain, l'esprit des trois conseils de pauvreté, chasteté, obéissance filiale à Dieu reconnu présent dans les supérieurs qui nous sont donnés. Avec les vertus solides, les dons du Saint-Esprit commencent à se manifester, surtout les trois moins élevés de crainte, de science et de piété. L'âme plus docile profite davantage des inspirations et illuminations intérieures.

Alors, si le progressant est vraiment généreux, commence d'ordinaire l'oraison infuse par des actes isolés de contemplation infuse au cours de l'oraison de recueillement; ${ }^{21}$

Avec les quelques variantes auxquelles il faut bien s'attendre, c'est un peu tout cet ordre de choses qui s'instaure en l'âme de Marguerite avec l'année 1640, et quoique au premier abord, les initiatives prises par celle-ci en la période 1640-1653, semblent bien diverses - jusqu'à sembler parfois incohérentes - sous l'apparent disparate des démarches effectuées, la jeune fille opère son unité intérieure à travers toute une série de purifications passives et actives où s'affine et se dépouille sa sensibilité dans le service de Dieu.

Consciente donc d'avoir été fortement touchée par l'Esprit-Saint, ${ }^{2} 2$ elle va tout d'abord se purifier aux pieds du grand pénitencier de Troyes, "Monsieur Daigarois ". ${ }^{2} 3$ Entrer dans la Congrégation Externe l'avait auparavant fait reculer, ${ }^{24}$ voici que faisant taire son respect humain elle s'y inscrit; ${ }^{25}$ de son propre aveu elle s'y montre fidèle congréganiste et en devient la préfète. ${ }^{26}$ Désireuse d'avancer

21. R. Garrigou-Lagrange, O.P. Les Trois Ages de la vie intérieure prélude de celle du ciel (Les Editions du Cerf, Paris, 1938) $1: 334$.

22. EA., 27, 126; - aussi Glandelet, VSMB., 4-5.

23. EA., 127.

24. Voir note 17.

25. EA., 127. - aussi Glandelet, VSMB., 5-6.

26. EA., 127: - aussi GLANDELET, VSMB., 9. 
spirituellement elle se met sous la direction de Monsieur Gendret, confesseur des Carmélites de Troyes ${ }^{27}$ qui va influer sur elle de façon décisive. Sur les conseils de ce prêtre qui lui parle de vie religieuse, elle va frapper à la porte du Carmel. Les moniales, a-t-elle remarqué:

me refusèrent quoyque je aves un grand panchant je fut a dautre mais cela ne reusy pas. ${ }^{28}$

Depuis longtemps Monsieur Gendret nourrissait un projet destiné à honorer la vie voyagère de la Vierge Marie suivant les apôtres dans leurs courses apostoliques après l'ascension de Jésus-Christ; en conséquence il rêvait de fonder une communauté de filles séculières qui, sans guimpe ni voile, seraient d'authentiques religieuses:

Ensuite Monsieur Jeandray me voulu bien prandre sous sa direction et me dit un jour que Notre Seigneur avait laissé 3 états de filles pour suivre et servir l'Eglise. Que celui de Ste Madeleine étoit rampli par les Carmélites et autre recluse, et celui de Ste Marte par les religieuses cloitrées qui servent le prochain, mais que celuy de la vie voiagere de la Ste Vierge qu'il falait honorer ne l'etoit; et que sans voile ni guimpe lon seroit vraiment religieuse ce qui métoit bien agreable car javois pitié des filles qui faute de bien ne pouvoient setablir au service de Dieu. ${ }^{29}$

Marguerite Bourgeoys éprise de perfection évangélique et docile instrument sous la direction de celui qui a autorité sur elle, donne la main au projet de communauté, qui échoue après une période d'essai dont nous ignorons la longueur exacte. ${ }^{30}$ Pour un observateur superficiel la jeune fille prenait de plus en plus les apparences d'une ratée dans la vie; en réalité celle-ci désormais située spirituellement dans la voie illuminative commençait de passer par le travail de la nuit des sens. Ces diverses déconvenues n'arrêtaient pas la marche en avant d'une âme qui désormais appartenait bien à Dieu. Le 21 décembre 1643, selon Glandelet, ${ }^{31}$ Marguerite émettait le vœu de chasteté et

27. EA., 127; - aussi Marguerite Bourgoys d M. Tronson, s.d., inséré dans EA., 28; - aussi GLAN DELET, VSMB., 6. EA., 28.

28. EA., 127; - aussi Marguerite Bourgeoys à M. Tronson, s.d., inséré dans

29. EA., 29, 106, 127; - aussi Glandelet, VSMB., 9.-10.

30. EA., 127; - aussi Glandelet, VSMB., 9-10.

31. VSMB., 8; - aussi EA., 141. 
puis, peu après, en 1644 peut-être, celui de pauvreté. En donnant ainsi à son effort spirituel la consécration de sa vie pauvre et chaste, elle ajoutait un trait de ressemblance avec les âmes saintes. En particulier, pour défendre sa chère pauvreté, elle aura toute sa vie durant, des accents et une ardeur qui ne sera pas sans analogie avec l'attitude de François d'Assise. ${ }^{32}$

Cependant le travail d'épuration que Dieu institue pour détacher du sensible ${ }^{3}{ }^{3}$ les âmes qu'il a marquées, se poursuivart en celle de Marguerite. Cette œuvre qui atteint tous les sens, Dieu entendait la continuer sous le coup des épreuves comme dans les joies qu'il distribuait dans la vie de sa fille. C'est ainsi qu'en l'année 1647, la jeune fille perdait son père qu'elle assista en sa dernière maladie et auquel elle rendit le difficile et dernier devoir de l'ensevelissement. ${ }^{34} \mathrm{La}$ sollicitude pour les mourants et la répugnante fonction de l'ensevelissement des morts, elle tiendra à l'exercer toute sa vie; dans la Champagne, sa patrie $;^{35}$ sur mer, au cours de ses traversées océaniques; ${ }^{36}$ et puis en terre canadienne. Enfin un peu plus tard, en la fête de l'Assomption de Marie, le 15 aout 1650, faveur inexprimable: Marguerite est en adoration devant le Saint Sacrement. ${ }^{37}$ Mue par l'Esprit elle lève les yeux vers l'hostie où elle aperçoit Jésus-Christ en la ravissante beauté de sa première enfance. De son propre aveu - et la chose a sa grande importance si l'on veut bien juger du travail d'épuration qu'opère en elle la nuit des sens - la voyante était:

sevrée pour toujours de toutes les beauté du monde. ${ }^{38-39}$

En mentionnant cette deuxième faveur ou apparition nous n'avons pas l'intention d'induire le lecteur en erreur: la vraie vie chrétienne ne réside pas dans ces charismes ${ }^{40}$ et il y aurait inconvénient grave à se méprendre sur ce point. Mais il reste vrai que sur l'une ou l'autre de ces

32. EA., 65, 95, 150: - aussi Glandelet, VSMB., 82-84.

33. A. TAN QUerey, Précis de théologie ascétique et mystique, 890.

34. Glandelet, VSMB., 11.

35. EA., 48.

36. Dollier de Casson, Histoire du Montréal, 101.

37. [E. Montgolfier ] La Vie de la Vénérable Saur Marguerite Bourgeoys, 45.

38.-39. Glan Delet, VSMB., 12; - aussi [ E. Montgolfier ] La vie de la Venérable Sœur Marguerite Bourgeoys, 45 . Il y a la, croyons-nous, exercice du don de science: " Par le don de science, en effet, s'explique le premier signe noté par SaintJean de la Croix: "aucune consolation dans les choses créées, ni dans les choses divines présentées d'une manière sensible ". Garrigod-Lagrange, Les Trois Ages de la vie intérieure, $2: 61$.

40. R. Garrigod-Lagrange, Les Trois Ages de la Vie intérieure, 2:747-790. 
faveurs extraordinaires (apparitions, paroles intérieures) s'est greffée pour Marguerite Bourgeoys son abnégation en face des biens créés et son union à Dieu, vrais facteurs, eux, de vie chrétienne.

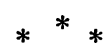

\section{$1652-1658$}

Toute proportion étant sauve la marche de l'âme en avant s'effectue de la même manière, qu'il s'agisse des commençants ou des progressants. D'un poin't de vue en effet - celui que nous allons signaler - vie purgative et vie illuminative s'identifient: dans les deux cas l'union à Dieu et le travail d'épuration qui y est essentiellement présupposé sont, d'économie tout au moins, lents, presque imperceptibles, marqués de retours en arrière et de reprises:

même les âmes avancées sont encore sujettes à beaucoup d'imperfections et sentent renaître en elles, sous une forme atténuée, les sept péchés capitaux... C'est pour les purifier et les préparer à un plus haut degré de contemplation que Dieu leur envoie différentes épreuves qui sont appelées passives, parce que c'est Dieu lui-même qui les produit et que l'âme n'a qu'à les accepter patiemment ${ }^{4}$.

C'est ce phénomène qu'il nous est loisible d'observer dans la vie de Marguerite Bourgeoys, particulièrement entre les années 1640 et 1670 , nous l'avons déjà remarqué. La phase de sa vie dans laquelle nous entrons présentement (1652-1658) n'apporte donc pas des phénomènes essentiellement différents de ceux déjà mentionnés. Tout simplement l'action épurante qui est celle de la Nuit des sens se poursuit; Dieu ne cesse pas de reprendre sans cesse le même travail chez sa fille bien que ce soit dans des circonstances concrètes toujours un peu différentes. Dans cette âme va ainsi se produire une œuvre d'affinement qui détruira progressivement les égoïsmes d'ordre sen-

41. A. Tanquerey, Précis de théologie ascétique et mystique, 890. 
sible pour opérer une certaine transfiguration de sa personnalité spirituelle:

Par nuit obscure nous entendons la Contemplation, et elle produit chez les spirituels deux genres de ténèbres ou de purifications, d'après qu'elle affecte l'un ou l'autre des deux éléments de l'homme, la partie sensitive ou la partie spirituelle. Il y a donc une première nuit ou purification des sens, qui donne à l'âme sa pureté en la dépouillant selon sa partie sensitive et en accommodant le sens à l'esprit. La seconde Nuit ou purification spirituelle est celle où l'âme se purifie et se dépouille selon l'esprit afin de l'accommoder et de la rendre apte à l'union d'amour avec Dieu. La Nuit des sens est rommune; elle se produit chez le grand nombre des commençants, et nous allons nous en occuper d'abord. La Nuit de l'esprit est exceptionnelle; elle est le privilège de ceux qui sont déjà exercés et avancés, et nous l'expliquerons en second lieu.

La première purification et Nuit est amère et redoutable pour le sens ainsi qu'on le verra. La seconde ne peut lui être comparée; elle n'est qu'horreur et épouvante pour l'esprit. ${ }^{42}$

Nous nous excusons de citer aussi longuement Saint-Jean-de-laCroix. Il nous a cependant paru essentiel de le faire afin qu'à travers les mille et un détails pris à même la vie de Marguerite ne soit pas perdue de vue par le lecteur la vérité qui fait trame. Ne pas garder ce lien suffisamment à vue peut aisément devenir ici l'écueil ${ }^{43}$. Des deux nuits ci-dessus mentionnées par le docteur de la vie spirituelle, ou, si l'on préfère, de cette double série de purifications, nous ne nous occupons dans cette seconde partie de notre travail (1640-1670) que de la première, celle des sens, ainsi que de la pratique héroïque des vertus chrétiennes qui lui est connexe. ${ }^{44} \mathrm{La}$ seconde nuit, celle de l'esprit n'ayant pas été le fait de Marguerite Bourgeoys avant l'année 1670, à ce que nous croyons, nous en renvoyons l'étude à la troisième partie (1670-1700).

1652 fut pour la Sœur Bourgeoys l'heure de la décisive entrevue avec Monsieur de Maisonneuve et de l'héroïque décision. Le gouver-

42. SAINT-JEAn DE LA Croix, La Nuit obscure et la vive flamme d'amour (Oeuvres complètes, Desclée De Brouwer, 1933), 3:27.

43. Gilbert J. Garraghan, A Guide to Historical Method, 404.

44. Garrigou-Lagrange, Les Trois Ages de la vie interieure, 2:581. 
neur de Montréal est alors de passage en France pour y lever la recrue d'hommes de laquelle dépend la survie de Ville-Marie. ${ }^{45}$ Les congrégantines de Troyes pressent celui-ci de les admettre en NouvelleFrance. ${ }^{45^{\mathrm{a}}}$

je moffre pour y aler et il macepte mais seule. Il parle à se père - (il s'agit de Monsieur Gendret) - et apres il par et etant a Paris il mande a sa sœur que si elle luy veu dire adieu quelle aille a Paris et quelle me mene avec elle ou elle le trouvera. Je parle a se bon pere et luy demande comme cela se fera que jaille en Canada sans autre conduitte de Monsieur de Maison neuve. Sa reponse fut: mettez vous sous la conduitte de cette personne comme entre les mains dun des premiers chevaillers de la chambre des Anges. ${ }^{46}$

Pour apercevoir toute l'héroïcité de cette décision, arrêtons-nous un moment à en peser les circonstances. La missionnaire n'ignore pas la situation critique de Ville-Marie ni surtout le massacre relativement récent des missionnaires jésuites survenu entre les années 1642 èt 1649 aux mains des Iroquois. M. de Maisonneuve n'a pu laisser ignorer ces choses aux Congrégantines qu'il écarte non plus qu'à la privilégiée. D'ailleurs depuis l'année 1632 les Relations sont lues et commentées en France ${ }^{47}$. Il n'est donc pas sans fondement de croire que lorsqu'elle s'offrit pour partir Marguerite Bourgeoys entrevoyait son apostolat en Nouvelle-France dans des perspectives de martyre possible. Si sublime que soit son geste, elle ne croit pas pour autant pouvoir se soustraire aux voies de l'obéissance. M. Gendret, M. Profit, son confesseur occasionnel, le Grand Vicaire de la cathédrale, M. Rose, sont tour à tour consultés ${ }^{48}$ et c'est de tels hommes qu'elle reçoit la décision finale comme des êtres providentiellement constitués sur elle.

Le moment est particulièrement solennel et l'appel à la sainteté se fait particulièrement exigeant pour elle; c'est pourquoi, semble-t-il,

45. F.-X. Garnead, Histoire du Canada (Editions de l'Arbre, 8e éd., Montréal, 1944), 2:53.

45a. EA., 28; - aussi Glandelet, VSMB., 14.

46. EA., 28, 128; - aussi Glan Delet, VSMB., 14-15.

47. Léon Pouliot, Etude sur les Relations des Jésuites de la Nouvelle-France (1632-1672) (Montréal, 1940), 16-17.

48. EA., 29; - Aussi Glandelet, VSMB., 14. 
le Ciel voulut manifester à l'héroïne une prédilection qu'elle n'avait certes pas escomptée. Peut-être restait-il encore quelques perplexités têtues en l'âme de la partante. Ou bien Dieu voulut-il tout simplement combler sa fille? Nous ne savons au juste. Toujours est-il que:

Un matin étant bien éveillée une grande femme vestue dune robe comme de serge blanche et me dit distintement va je ne te delaisseray point et je connu que setoit la Ste Vierge ce qui me donna bien du courage et je ne trouves rien de difisile quoy que je cregnes les ilusions ce qui me fit panse que si cela etoit de Dieu que je n'avois que faire de rien pour cela qui fit que je naportay pas un denier pour mon voyage et dans ses temps depuis ma touche jai ete toujours conduitte par ce chemin de perfection quoy que je ny ay pas profite mais dans toutes les rancontres jay toujours promis a Dieu de faire mon posible pour y arive avec le secours de sa grace..$^{49}$

Force surnaturelle, confiance en Dieu, défiance à l'égard d'ellemême, pauvreté et humilité constituèrent donc l'atmosphère dans laquelle s'effectua l'héroïque départ de Troyes. Avec une simplicité qui tient du sublime la missionnaire ajoute:

Je pardt sans denier ni maille un petit paquet que je pouvois porter sous mon bra..$^{50}$

Le passage à Paris marqua spirituellement. Tout d'abord elle fit cession de ses droits à l'héritage paternel en faveur d'un frère et d'une sœur plus jeunes. Et puis elle tomba dans un désarroi profond. Sans compter les oppositions qu'il lui fallut affronter de la part de l'oncle Cossard irrité, ${ }^{51}$ la tentation de la vie carmélitaine revint sous une forme des plus spécieuses: Marguerite logeait chez une personne de qualité, Mademoiselle de Bellevue, dont le frère était le provincial des Carmes pour la région de Paris. Celui-ci:

me fit dire, note-t-elle, que si je voules estre Carmelite je seres ou je voudres comme jy aves postule a Troyes. ${ }^{52}$

Une offre de cette nature semblait remettre en question tout le problème. On se rappelle en effet la grande inclination qu'éprouvait

49. EA., 129; - aussi Glan delet, VSMB., 15.

50. EA., 29, 129; - aussi Glandelet, VSMB., 16.

51. Glan delet, VSMB., 16.

52. EA., 29; - aussi Glandelet, VSMB., 17. 
Marguerite pour l'idéal carmélitain ${ }^{5}{ }^{3}$ et l'on comprend aisément que la pauvre fille ait été ébranlée. Une fois de plus la soupape d'échappement fut l'obéissance; un jésuite consulté - qui connaissait le Canada - la maintint dans son dessein de vie missionnaire. ${ }^{54}$

L'itinéraire Paris-Nantes était affaire de quelques jours; ils suffirent pour donner à la voyageuse l'occasion de beaucoup souffrir: promiscuité du coche et des hôtelleries, propositions de nature équivoque, soupçons entretenus par les gens honnêtes, etc., rien ne lui fut épargné. ${ }^{5}$ Des avanies semblables marquèrent le séjour à Nantes où, entre autres choses, Madame Le Coq crut d'abord flairer en elle une aventurière. ${ }^{56}$ Enfin, et voici le comble: une indiscrète insistance du Père Carme et puis des lettres anonymes lui remirent en l'esprit la tentation de Paris. De nouveau, obscurité, détresse d'une âme timorée qui ne put retrouver la paix intérieure qu'au pied du SaintSacrement en l'Eglise des Capucins. ${ }^{57}$

On sait en quelles circonstances lamentables s'effectua la traversée de 1653 sous la conduite de M. de Maisonneuve. D'arrache-pied, celuici était parvenu à recruter quelque 108 hommes pour Ville-Marie; deux vaisseaux successivement employés, dont le premier était avarié et le second infecté; obligation de relâcher à 350 lieues en mer; mutinerie de la recrue; enfin à bord, la peste à l'état épidémique et huit hommes fauchés par la mort. ${ }^{58}$ Marguerite Bourgeoys se fit infirmière. Tout le malade, corps et âme, devint l'objet de sa sollicitude. Pendant de longues semaines, elle se fit la providence des pestiférés, le prêtre des agonisants, l'ensevelisseuse qui rend aux morts les derniers devoirs. Et lorsque le 22 septembre de cette mémorable année 1653 le navire se montra en vue de Québec, la charitable infirmière avait encore des malades confiés à ses soins. ${ }^{59}$

53. Voir note 28.

54. EA., 29; - aussi Glan delet, VSMB., 17.

55. M.-F. Ransonnet, Vie de la Sœur Marguerite Bourgeoys (Liège, 1728; copie manuscrite déposée aux ACNDM.), 4.

56. C. Glandelet, VSMB., 19; - [ E. Montgolfier ], La Vie de la Vénérable Sour Marguerite Bourgeoys, 59.

57. EA., 30;130; - Glan delet, VSMB., 20.

58. EA., 38.

59. EA., 38. 
M. de Maisonneuve était encore sous le coup de l'admiration que lui avait inspirée durant la traversée l'attitude héroïquement charitable de la Sœur lorsque, à peine débarqué à Québec, il formulait à Jeanne Mance son jugement dans les termes suivants:

une bonne fille que j'amene nommée Marguerite Bourgeoys dont la vertu est un trésor qui sera un puissant secours au Montreal, au reste cette fille est encore un fruit de notre Champagne qui semble vouloir donner à ce lieu plus que toutes les autres réunies ensemble. ${ }^{60}$

Celle que visait ce témoignage n'était pas sans l'avoir mérité de multiples façons. Nous en avons déjà relevé quelques-unes; il convient d'en mentionner d'autres pour que soit rendue manifeste l'inspiration éminemment spirituelle de cette vie. Tout d'abord l'influence christianisante de la Sœur sur les hommes de la recrue. On se rappelle la réflexion faite par Marguerite Bourgeoys sur le danger couru lorsque à 350 lieues en mer, le Saint-Nicolas donnant de la bande et faisant eau plus qu'on en pouvait tirer, avait dû revenir à SaintNazaire:

en approchant nous perisions sans le secour que par la grace de Dieu nous eusmes de ce lieu la de quoy setoit fort en penne car nous etions pres de 120 passagers sans prestres et les 108 soldas mal preparés pour mourir ausi bien que tout le reste... ils etes comme des furieux et croies quon les menoit en perdition. ${ }^{6} 1$

Voici par contraste le témoignage que la Sœur juge bon de rendre à ces mêmes hommes dès leur arrivée à Québec le 22 septembre (1653):

Melle Mance retourna à Montréal et je restai seule à Québec pour faire fournir les provisions aux soldats. Ils étaient doux comme de vrais religieux, ce qui me donnait bien de la joie d'aller avec eux à Ville-Marie; et peu de temps après leur arrivée dans ce lieu, ces cent hommes étaient changés comme le linge qu'on a mis à la lessive. ${ }^{62}$

La Sœur omet de dire que dans l'intervalle il y avait eu l'épidémie à bord, l'influence conquérante de l'infirmière et sa maternelle charité par laquelle ces rudes hommes avaient été gagnés.

60. Dollier de Casson, Histoire du Montréal, 100.

61. EA., 39.

62. EA., 50. 
Lorsqu'en novembre seulement de la même année (1653) Marguerite Bourgeoys met le pied dans Ville-Marie, le terme ultime de son voyage, c'est, on s'en souvient, pour enseigner les enfants. En fait quatre longues années vont se passer avant qu'elle dispose d'une école et d'une recrue d'élèves méritant ce nom. Durant ce laps de temps que fera-t-elle donc? Elle ne va pas cesser de répondre aux volontés divines sur elle-même au fur et à mesure que se manifesteront celles-ci; et ce n'est pas là certes l'aspect le moins intéressant de cette grande vie. Sans aucun doute la première éducatrice de Ville-Marie a réalisé de grandes choses; parfois même l'extraordinaire et le miraculeux sont venus scander ses initiatives. Il n'empêche que là n'est pas le plus beau. Surnaturellement fixée sur la nature de sa mission en Nouvelle-France elle n'a procédé, toutefois, dans l'exécution de son mandat que dans les limites de l'obéissance et n'a donné pleine adhésion aux mots d'ordre venus de Dieu que dans la voie tout ordinaire de la soumission à l'autorité; c'est à ce niveau seulement que se laisse apercevoir l'unité vraie et profonde de cette vie.

Nombreux sont les cas qui s'inspirent de cette attitude spirituelle. Lorsque Marguerite Bourgeoys arrive au Canada, c'est pour y faire œuvre d'éducatrice; mais dans le Ville-Marie d'alors, point d'enfants en état d'apprendre. La pénurie de mariages conclus depuis la fondation du poste (1642) et surtout la mortalité infantile a créé cet état de choses:

On a été environ huit ans, dit la Sœur Bourgeoys, sans pouvoir garder d'enfants à Montréal; ce qui donnait bonne espérance puisque Dieu prenait les prémices. ${ }^{63}$

A l'interprétation surnaturelle qu'elle donne de cette situation poignante, J'on peut voir que la vie intérieure de la Sœur Bourgeoys n'est pas un épiphénomène. S'il en était autrement elle ne pourrait apercevoir ainsi dans les perspectives de la foi une épreuve capable de prendre pour Ville-Marie une si grave signification. A Dieu ne plaise, cependant, que la Sœur se consume en de stériles regrets; en attendant la gent écolière elle sera la bonne à tout faire de l'établissement de Montréal: ménagère dans la maison du gouverneur; ${ }^{64}$ maman

63. EA., cité dans E. FAILlon, Vie de la Sœur Bourgeoys, 1:78.

64. La Sœur Morin, Annales de l'Hôtel-Dieu de Montréal (Mémoires de la société historique de Montréal, 12e livraison, L'imprimerie des Editeurs Limitée, Montréal, 1921) 85. 
d'adoption pour les petits comme Marie Dumesnil et Jeanne Loisel; ${ }^{65}$ soutien moral de Jeanne Mance; ${ }^{66}$ aviseuse spirituelle de M. de Maisonneuve; ${ }^{67}$ pourvoyeuse infiniment charitable des soldats dans le froid hiver de $1653-1654 ;{ }^{68}$ architecte improvisée du sanctuaire de Notre Dame de Bon-Secours; ${ }^{6} 9$ enfin mandatée de M. de Maisonneuve pour restaurer la Croix en la Montagne ${ }^{70}$. Marguerite Bourgeoys vient prendre rang dès son arrivée en Nouvelle-France à côté de Maisonneuve, de Jeanne-Mance; elle s'affirme comme l'un ou l'autre de ces êtres providentiels au sens chrétien et à l'industrie desquels le Montréal primitif dut de survivre aux heures les plus critiques de son passé. ${ }^{70^{\mathrm{a}}}$

Dans une circonstance qui le mettait personnellement en cause et où il lui fut donné de franchir une importante étape de sa propre vie intérieure, le gouverneur de Ville-Marie put juger de quelle trempe spirituelle était la jeune recrue de Troyes. L'événement dont nous voulons parler se situe entre les années 1b53, date d'arrivée au Canada pour la Sœur et 1655, alors que M. de Maisonneuve retraverse la mer pour aller demander des prêtres à Monsieur Olier. Le gouverneur de Montréal avait quelque chose du moine-soldat: courageux dans le combat, de commandement judicieux autant que ferme, pieux et donnant à ses colons l'exemple de la vie chrétienne, Paul de Chomedey de Maisonneuve était un célibataire austère et chaste qui, cependant, n'échappa pas à la lutte. Avec humilité il s'en ouvrit un jour à l'un des pères Jésuites qui desservait la colonie. Celui-ci lui conseilla le mariage. Mal satisfait de cette décision, il ne crut pas déchoir en pre-

65. EA., 37, 50 .

66. Dollier de Casson, Histoire du Montréal, 101.

67. La Sœur Morin, Annales de l'Hôtel-Dieu de Montréal, 84.

68. [ E. Montgolfier] La Vie de la Vénérable Saur Marguerite Bourgeoys, 66-67.

69. La Sœur Morin, Annales de l'Hôtel-Dieu de Montréal, 85.

70. EA. 33.

70a. M. le Chanoine Groulx a campé le personnage de Marguerite Bourgeoys lorsqu'il a écrit des fondateurs de Montréal les lignes suivantes: "Nos fondateurs nous apparaissent grands, non seulement par le labeur qu'ils mettent dans leur œuvre, labeur effrayant, mais aussi et peut-être plus, par les lointaines visées qu'ils y renferment. Parce qu'ils travaillent pour un long avenir, tous les jours ils apprennent a se dépasser. "Notre Mâ̂tre, le Passé (Bibliothèque de l'Action Française, Montréal, 1924), 32. 
nant avis de la Sœur Bourgeoys dont la solution fut diamétralement opposée: émettre le vœu de chasteté était selon elle la mesure à prendre. Le gouverneur y souscrivit après approbation du Père Jérôme Lallemant et l'âme du chrétien s'en trouva pacifiée. ${ }^{71}$

Il nous semble, sauf erreur, qu'en conseillant ainsi le vœu de chasteté à M. de Maisonneuve, Marguerite Bourgeoys usa - probablement sous l'influence du don de conseil - d'une prudence supérieure, prudence à laquelle nous voyons que l'Esprit-Saint lui donna maintes fois accès durant sa vie. On sait d'ailleurs que la vie illuminative dans laquelle évoluait la Sœur depuis la conversion de 1640 comporte l'exercice plus fréquent et plus caractérisé des dons. ${ }^{72}$

Pour simple mention, notons enfin deux autres entreprises dont la conduite fut confiée à la Sœur en cette époque: la restauration de la Croix en la Montagne et l'érection du sanctuaire de Bon-Secours. Ces deux œuvres témoignent, chacune à leur façon, de la force morale et du profond esprit de religion existant au cœur de la cheftaine; et il n'est que juste de relever quelques incidents dans le récit des Écrits Autographes pour saisir la nature des dangers et difficultés auxquels elle dut faire face: un détachement de pas moins de 30 hommes fut jugé nécessaire pour garantir la sécurité des ouvriers-pèlerins dans la montagne; la raison en était que la Croix était l'enjeu d'une lutte:

Mr de Maisonneufve detacha 30 hommes pour saquiter de la promesse qu'il mavoit faite de me mener sur la montagne mais les sauvages aves oté la croix. On a exité le monde a y en rebatir une nouvelle. je fit destinée pour cela. ${ }^{73}$

Trois jours de suite on prit les mêmes risques et la croix fut érigée de nouveau, mais - et c'est ce qui établit qu'excursionner dans $s_{\text {in }}^{\alpha}$ la montagne à cette époque avait ses dangers-:

l'on ne put plus y retourner; car il survint des empêchements de la part des iroquois qui se cachaient dans les bois pour surprendre nos travailleurs. ${ }^{74}$

Quant à l'érection du sanctuaire de Bon-Secours elle eut quelque chose d'une création tant les moyens dont on disposait dans l'état

71. Voir note 67.

72. R. Garrigod-Lagrange, Les Trois Ages de la Vie intérieure, 2:60.

73. EA., 33.

74. Ibid. 
précaire où gisait alors le poste de Ville-Marie paraissaient humainement rudimentaires: ils se ramenaient en fin de compte à la foi et à la bonne volonté des colons du poste:

j'exite, à écrit la Sœur Bourgeoys, le peut de personne a ramaser des pierre et $M$ de $M$ - [M. de Maisonneuve]- fit couper du bois pour la charpante et il aidoit a le trener hors du bois et ceux a qui je faises quelque travail je leur demandes quelque journée pour cette chapelle. On charea du sable et les masons sofre dy travailler. Le père Picar la nomme Notre Dame de Bon Secours. Le Pere Lemoine met la premiere pierre Monsieur Clause fait gravé sur une lame de cruivre avec l'inscription nesaisere et les masons commance. ${ }^{75}$

Nous verrons un peu plus loin, dans la troisième partie de cette étude, comment en réaction pour cette charité, la Vierge de BonSecours vint en aide à sa fille lorsque celle-ci, poursuivant son crucifiant itinéraire spirituel, entra pour de bon dans la Nuit de l'Esprit aux abords de l'année $1670 .^{76}$

$1658-1670$

$\mathrm{Au}$ nombre des obligations assumées par la Compagnie des Messieurs et Dames de Montréal figurait celle-ci: créer un séminaire de religieuses pour instruire les filles à la française. C'est à satisfaire à cette obligation que tendait l'initiative prise en 1658 par M. de Maisonneuve concernant Marguerite Bourgeoys:

4 ans apres mon arivée Monsieur de Maison neufve me voulu donne une étable de pierre pour faire une maison pour logé celle qui y feres l'école. Jy fit faire une cheminée et ce qui etoit necessoire pour y loger les anfants la curerent et y travaillerent en tout se quils pouves. ${ }^{77-78}$

A ne lire que dans leur matérialité ces lignes que la Sœur écrivit - comme du reste la plus grande partie de ses mémoires - abstrac-

75. EA., 47; - aussi Glandelet, VSMB., 26.

76. EA., cité dans FaIllon, Vie de la Sœur Bourgeoys, $1: 205$.

77-78. EA., 40; - aussi Glandelet, VSMB., 24-25. 
tion faite de ses dispositions affectives, on imaginerait difficilement la joie qu'elle ressentit en inaugurant, surtout dans ces conditions de dénuement, son œuvre éducative. Cependant elle en éprouva un profond bonheur. Tout d'abord elle touchait le but pour lequel elle avait quitté sa patrie, et surtout elle débutait comme éducatrice dans une pauvreté qui n'était pas sans analogie avec l'apparition de Jésus sur la terre dans l'étable de Bethléem. Qu'elle ait profondément chéri cette pauvreté ne fait aucun doute pour quiconque pense aux misères, aux scrupules qu'elle se créera plus tard pour avoir autorisé en 1663, sous la pression de ses sœurs, la construction d'une "spacieuse " maison de pierre. ${ }^{79}$ Comme on s'en doute un peu, le nouvel immeuble n'était rien moins que luxueux; il n'empêche que la Sœur y verra toujours quelque chose comme la trahison de son idéal de pauvreté, idéal que seule l'étable de la Commune incarnait à son gré. 80

Dans l'accomplissement de sa nouvelle fonction, Marguerite allait apporter ce qu'elle possédait, comme fille du peuple, de formation intellectuelle et morale. Elle allait surtout fournir à l'enfance de VilleMarie l'apport de son irremplaçable expérience de la vie chrétienne. A ne regarder que ce qu'elle apportait de connaissances strictement profanes, le bagage de l'éducatrice peut paraître mince; ${ }^{81}$ mais pour quiconque ne perd point de vue la situation concrète dans laquelle le colon de Ville-Marie devait vivre en cette deuxième moitié de dixseptième siècle, il est manifeste que l'éducatrice avait précisément de quoi répondre aux besoins de l'heure.

L'école s'épanouit très vite en des œuvres complémentaires qu'exigeaient impérieusement les indigences de Ville-Marie. Des mioches comme Adrienne Barbier, Nicolas Desroches, Jean Leduc, François Loisel et Catherine Daubigeon n'étaient pas encore mûrs pour le travail scolaire proprement dit; à l'instigation de la fondatrice l'école se fit pour eux pouponnière et jardin d'enfance. ${ }^{82} \mathrm{D}$ 'autre

79. Glandelet, VSMB., 25.

80. Ibid.

81. M. Montgolfier observe qu'elle " apprit à lire et à écrire, et peut-être quelque chose de plus: mais surtout les vrais principes de la piété chrétienne. "La Vie de la Venérable Sceur Marguerite Bourgeoys, 36. Cela pourrait bien être la note juste; en effet Marguerite était fille et, qui plus est, fille du peuple. Dans la France du XVIIe siècle ces deux qualifications mises ensemble ne postulaient pas généralement une instruction bien poussée.

82. Ainsi A. JАMET, Marguerite Bourgeoys, 1:185. 
part, Marie des Neiges, le poupon de l'iroquoise Teonnetharay, fut la première privilégiée du séminaire que la charité de Marguerite Bourgeoys décidait d'offrir aux petits enfants des sauvages. ${ }^{8}{ }^{3}$ Enfin, toujours en l'année 1658, le 2 juillet cette fois-ci, la missionnaire se donna une troisième tâche dans le champ de l'éducation chrétienne; et cette nouvelle initiative témoigne de l'ampleur de vues avec laquelle l'institutrice considérait sa vocation en Nouvelle-France. Pour les adolescentes de Ville-Marie, déjà épouses ou bien en passe de l'être, elle créa une congrégation externe à l'image de celle dont elle avait été la préfète à Troyes avant l'année $1653 .{ }^{84}$ Dans la pensée ce nouvel organisme de vie sociale et chrétienne devait venir en aide à l'enfant que la dureté des temps engageait très tôt en qualité d'épouse et de mère dans les devoirs de la vie conjugale et familiale. Il devait affermir ces adolescentes dans la pratique de la vie chrétienne, leur donner cette maturité d'âme exigée par leur maternité précoce, aussi peut-être les entraîner à la bonne tenue matérielle d'un foyer.

Sur l'œuvre initiale de l'école sont donc venues se greffer très tôt des œuvres annexes qui tendaient toutes à préparer ou à parfaire l'éducation de l'enfant ainsi qu'à façonner les épouses et les mères chrétiennes dont Ville-Marie avait le plus pressant besoin. Et ceci nous amène à faire la double observation suivante: tout d'abord, en agir ainsi supposait chez Marguerite Bourgeoys l'existence d'un patriotisme authentiquement chrétien. ${ }^{85}$ Et puis, si incontestablement la Sœur n'est pas une contemplative pouvant aller de pair avec Marie de l'Incarnation - encore qu'elle ait eu pour partage une certaine contemplation ${ }^{86}$ - en revanche la tendance interne de sa vie chrétienne était principalement l'action, l'action apostolique et, d'une façon plus précise encore, l'action éducatrice. Avant que d'être une contemplative, la première maîtresse d'école à Ville-Marie a été une femme d'œuvres, et c'est pourquoi, pensons-nous, sa personnalité spirituelle prend forme et consistance plus par la mise en œuvre des vertus et

83. EA., 41-42.

84. EA., cité dans E. Falllon, Vie de la Sœur Bourgeoys, 1195.

85. EA., 75-76.

86. On sait que selon la doctrine de Saint-Jean de la Croix, la vie illuminative et plus encore la vie unitive ne va pas sans un certain degré de contemplation d'ailleurs variable avec les diverses étapes de la vie spirituelle. 
des dons à finalité active (prudence, conseil et force) que par l'exercice des habitus de contemplation comme les dons de sagesse et d'intelligence.

Décidément l'année 1658 avait marqué. La vocation d'éducatrice pour laquelle Marguerite Bourgeoys était passée en Nouvelle-France avait pris des modalités concrètes. Avec l'apparition de l'école de la Commune ainsi que des œuvres complémentaires, (le jardin d'enfance, le séminaire pour les enfants sauvages et la congrégation externe) l'Oeuvre de la missionnaire avait commencé de prendre consistance et aussi une relative ampleur. C'était beau; mais aussi c'était beaucoup pour les forces d'une seule femme, même passagèrement aidée par Marguerite Picar. La Sœur Bourgeoys décida d'aller chercher des recrues en France. Une occasion providentielle s'offrait pour cela. Depuis plus d'une année déjà Jeanne Mance s'était rompu le bras droit et démis le poignet (28 janvier 1657), et depuis ce temps l'estropiée s'était vue pratiquement dans l'impossibilité d'exercer ses fonctions d'hospitalière. ${ }^{87}$ Deux raisons la poussaient donc à faire la traversée: obtenir que les Hospitalières de La Flèche vinssent prendre la direction de son hôpital et puis consulter les chirurgiens de France.

5 ans apres mon arivee a Montreal Mademoiselle Mance qui avoit le bra rompu avoit besoin dune personne pour la soulager je mofre pour cela afin daler a Troyes pour avoir quelque filles pour m'aider a faire lecolle. ${ }^{88}$

Charité pour Jeanne Mance et providence sur son œuvre furent donc les deux mobiles de la voyageuse. Elles partirent toutes deux le 29 septembre (1658). ${ }^{8}{ }^{9} \mathrm{Il}$ est à remarquer qu'en allant se recruter des collaboratrices auxquelles elle va essayer par la suite d'insuffler son esprit, la missionnaire paraît bien jeter les premiers fondements de sa communauté. Dans quelle mesure est-elle consciente, à cette époque, de son rôle de fondatrice? Dieu seul le sait. Pour ce qui est de nous, nous ne saurions le dire avec certitude, car l'historien Glandelet a écrit à ce sujet des lignes qui ne peuvent que laisser perplexe quiconque les lit et les pèse:

Elle n'avait pourtant en venant au Canada, a noté l'historien, aucun dessein d'y établir une communauté comme elle l'avoue

87. EA., 42.

88. Ibid.,

89. EA., cité dans E. FAILlon, Vie de la Sœur Bourgeoys, 1:129. 
elle-même dans l'un de ses écrits; et même les filles qu'elle amena six ans après, de Troyes en France où elle avait repassé pour la première fois, n'étaient engagées que par contrat civil pour demeurer ensemble et faire avec elle les fonctions de maitresse d'école à Montréal, sans penser pour lors à y faire aucun établissement dans les formes. Ce dessein ne s'est formé à proprement parler que lorsqu'elle fit son second voyage en France, en mil-six-cent-soixante-dix..$^{90}$

Le cas n'est pas rare dans l'histoire des spirituels, d'âmes que durant un certain laps de temps Dieu a maintenues dans une partielle inconscience d'elles-mêmes. Quoi qu'il en puisse être de Marguerite Bourgeoys, consciente ou non de son rôle de fondatrice, elle accomplit dès les années 1658-1659 des gestes qui en eux-mêmes et à des degrés divers ont valeur de fondation.

De ce premier retour en France nous ne fixerons que quelques détails strictement indispensables si nous ne voulons pas perdre de vue la vie intérieure de la voyageuse. Tout d'abord une traversée de quelque deux mois, période de souffrances pour la directrice de l'HôtelDieu et de charité effective pour l'infirmière bénévole, période de sacrifices et de privations pour les deux qui restent confinées, notent les Écrits autographes, dans la chambre aux canons. ${ }^{91}$ Arrivées à Paris dans la deuxième partie de janvier 1659, elles se séparent entre le 28 de ce mois et le 1er février; et pendant que Jeanne Mance après avoir entendu les chirurgiens de Paris prononcer leur inexorable verdict sur son cas se voit miraculeusement guérie par l'intercession de Monsieur Olier, ${ }^{9} 2$ Marguerite Bourgeoys prend la route de Troyes où elle reçoit l'hospitalité de ses amies, les Congrégantines. Là elle procède au travail de recrutement pour lequel elle est venue et groupe autour d'elle ses trois premières compagnes, Catherine Crolo, Marie Raisin et Edmée Chatel. ${ }^{93}$ Les circonstances dans lesquelles s'opéra la conquête de cette dernière sont particulièrement révélatrices de la vie intérieure de la fondatrice: une fois de plus son idéal de pauvreté héroïque miroite à nos yeux:

Au premier voiage que j'ai été en France jay admiré comme Monsieur Chatel notaire apostolique ma confié sa fille quil

90. Glandelet, VSMB., 3.

91. EA., 45.

92. EA., 42.

93. EA., 43. 
aimoit beaucoup ne voiant rien pour sussister logee en une table ou javes fait faire une cheminee et quelque retranchement. Cette table avoit servie de colomber et de loge des bestes a cornes ou il faloit monte par une echele par dehors pour couché dans le grenier toutes de ran et quand se Monsieur me dit de quoy nous vivrions je luy promis que aurions du pain et du potage et que nous travailleront pour gaigner notre vie se qui le fit pleurer. ${ }^{94}$.

La réponse de Marguerite au notaire apostolique tient du sublime; elle rappelle le mot de Saint Paul montrant ses mains aux anciens de l'Eglise d'Ephèse et disant non sans quelque fierté apostolique: " ces mains ont pourvu à mes besoins ). ${ }^{95-96}$

C'était une petite caravane que vers la fin de février (1659) la Sœur dirigea sur Paris - quinze ou vingt personnes - et le voyage de même que l'étape suivante, Paris-Larochelle, fut franchi au milieu de mille tracasseries et misères. Au port d'embarquement en particulier les mesquineries des hommes et les perplexités de la Sœur furent telles qu'elle laissa échapper un gémissement significatif chez cette femme forte qui avait pourtant la plainte ni fréquente ni facile:

Etant a La Rochelle on nous avoit promis quon nous ambarqueroit pour chacune 50 livres et nous navions point dargent. On refuse Monsieur de Maison neufve pour repondant on veut que ma Sœur Raisin san retourne pour faire paier en France. Me voila bien en peine. ${ }^{97-98}$

Cette troisième traversée fut marquée par le même fléau que la première. Le Saint-André qui avait précédemment servi de navire-hôpital dans l'une ou l'autre des guerres du royaume, était encore infecté vraisemblablement. La peste s'y déclara; ${ }^{9}{ }^{9}$ elle épargna peu de passagers et le bilan des deuils, des dévouements et des conversions ne fut pas moindre qu'en 1653; deux huguenots abjurent l'hérésie avant de mourir; Jeanne Mance est dangereusement atteinte pendant que $\mathbf{M}$. le Maistre, l'un des deux ecclésiastiques de M. Olier à bord, se prodigue auprès des mourants et ensevelit les morts. Marguerite Bourgeoys,

94. EA., 43, 50.

95-96. Actes des Apôtres, $20: 34$.

97-98. EA., 51.

99. EA., 45, 146. 
quoique souffrant elle aussi du mal commun, redevient l'infirmière de jadis et soustrait à la mort l'enfant des Thibodeau que, dans la panique générale, d'aucuns voulaient jeter à la mer. ${ }^{100}$ Mais en définitive qu'importent toutes ces misères et avanies; la Sœur Bourgeoys sait d'expérience que la conduite des œuvres de Dieu ici-bas est chose austère, et cela, elle est femme à ne pas l'oublier alors qu'elle est à la veille de débarquer au pays avec une première équipe d'apôtres qui seront dès demain les Institutrices de Ville-Marie.

Qu'on n'imagine pas du reste chez elle une vie spirituelle étroitement refermée sur elle-même ou sur la besogne d'éducatrice, sans sollicitude véritable pour les intérêts de Ville-Marie et du pays tout entier. La réalité était autre. Avec fierté et pitié tout à la fois elle consigne dans les Écrits Autographes le glorieux fait d'armes du Long Saut (1660), ${ }^{101}$ les martyres de MM. le Maistre, Saint-Père, Brigeart; ${ }^{102}$ le massacre des hurons chrétiens par les perfides Iroquois; ${ }^{103}$ elle devient, avec la mère de Brésole, le réconfort moral de M. de Maisonneuve lorsque commencent les difficultés avec $M$. de Mésy; ${ }^{104}$ enfin, en vraie sociétaire du Corps mystique de Jésus-Christ, elle aperçoit dans les Hospitalières de Jeanne Mance, des âmes sœurs auxquelles elle lie spirituellement ses institutrices.

Cette même période qui suivit le retour de France marqua de nouveaux développements pour l'œuvre de Mère Bourgeoys. En l'année 1663 (peut être 1664) celle-ci qui avait déjà pourvu d'un foyer six ou sept filles à marier de la recrue de 1659, dut répéter son geste en faveur d'un nouveau contingent, plus fort cette fois, de futures épouses. Elle le fit avec toute sa charité, mais en même temps elle accomplissait un geste qui, avec d'autres, devait devenir dans la dernière période de sa vie, la source de fortes peines intérieures et de crucifiantes purifications:

Quelques anée apres il arive anviron 17 filles du Roy que jale querir au bor de leaux croiant quil faloit ouvrir la porte de la maison de la Ste Vierge a toute les filles notre maison etoit

100. EA., 45-46.

101. EA., cité dans Faillon, Vie de la Sceur Bourgeoys, 1:145.

102. EA., 34.

103. EA., 33.

104. La Sœur Monin, Annales de l'Hotel-Dieu de Montréal, 81. 
petitte nous fimes accomoder la petite maison achetée de StAnge et je demeres avec eux et jetes obligée dy demeurer a cause que s'était pour faire des familles $J_{\Theta}$ crois que je ne faisois pas plaisir à nos sœurs et que je manques a leur donner les instructions nesaieres. ${ }^{105}$

Trois choses sont à noter dans ce témoignage: la charité dont fait preuve Marguerite Bourgeoys pour ces enfants sans mère, le délaissement relatif et momentané auquel elle abandonne ses compagnes et surtout le malaise qui commence d'en résulter pour l'équipe des éducatrices. Il n'était pas possible que la Congrégation naissante échappât aux vicissitudes qui marquent toutes les entreprises humaines; et ce malaise presque insignifiant aux débuts, préludait quand même aux difficultés plus graves que la fondatrice allait connaître dans le gouvernement de son groupe. Nous voulons parler de la défiance que la fondatrice commença à éprouver vis à vis d'elle-même à partir de cette époque et du sentiment d'infidélité à Dieu dans l'accomplissement de son devoir d'état qui lui devint vite un véritable tourment. C'est ainsi, croyons-nous, qu'avec l'année 1663, Marguerite Bourgeoys s'oriente d'ores et déjà vers la Nuit de l'esprit, cette période de ténèbres et de désolations dans laquelle elle va passer les trente dernières années de sa vie 1670-1700 et qui constituera le sommet de sa vie spirituelle sur la terre.

De nouveau en l'année 1663, des divergences de vue se produisirent entre la fondatrice et ses sœurs: en dépit de l'achat fait précédemment de la concession Saint-Ange, la Congrégation étouffait dans ses deux étables. Il était donc impérieux pour elle de se mettre un peu au large. Les compagnes en tenaient pour une spacieuse maison de pierre que l'on construirait un peu plus haut que la commune et encore à proximité de l'Hôtel-Dieu; Marguerite Bourgeoys, elle, répugnait à échanger le Bethléem des débuts contre ce qu'elle entrevoyait comme une luxueuse habitation. Sa chère pauvreté, pensait-elle, y serait sacrifiée..$^{106}$ Néanmoins elle céda à ses sœurs, mais ce fut en se le reprochant comme une faiblesse et en regrettant de se mettre par là dans l'impossibilité d'édifier le Sanctuaire de Bon-Secours dont la construction avait dû être abandonnée sitôt que commençée, après l'arrivée de $M$. de

105. EA., 146-147.

106. EA., 147. 
Queylus au Canada (1657). Quoi qu'il en soit en cédant à la pression exercée par ses sœurs, la fondatrice prenait une décision qui allait rendre plus douloureux encore l'état d'âme dont nous venons de parler.

L'année 1669 marqua un bon moment dans la vie de la fondatrice; peut-être lui apporta-t-il un allégement momentané de ses peines: le 20 mai Mgr de Laval donnait à l'Institut de la Congrégation un mandat qui l'authentiquait pour la colonie entière. Une sanction de cette qualité était de nature à redonner confiance à celle dont les initiatives devenaient ainsi l'objet d'une publique approbation de la part du chef qui, neuf années auparavant, avait transmis à Rome un jugement louangeur sur les Institutrices de Ville-Marie.

Cet événement heureux n'empêchait pas Dieu, en ces mêmes années, de maintenir la fondatrice dans les aspérités de la vie spirituelle. A ce point de vue l'année 1670 ne devait pas être sans fardeaux. Il y eut tout d'abord la tentative faite par les colons de Ville-Marie pour attirer les Ursulines dans leurs murs. De plus la Sœur continuait de souffrir de deux choses: nous avons noté plus haut le malaise qui s'était glissé entre elle-même et ses sœurs lorsque, aux environs de 16631664, elle avait quelque peu délaissé celles-ci pour demeurer avec les dix-sept filles du roi; de ce malaise il demeurait encore quelque chose, semble-t-il, en 1670. Et puis la Sœur se reprochait toujours comme une infidélité la construction de la "luxueuse " maison de pierre dont les travaux maintenant avancés l'avait empêchée de construire le Sanctuaire de Bon-Secours. Depuis l'année 1663 le malaise intérieur avait augmenté jusqu'à devenir d'intolérables peines d'esprit dont la pauvre âme gémissait parfois:

Depuis mon retour de France, a-t-elle noté, - (il s'agit de la traversée de 1659) - étant en communauté avec mes sœurs je n'avais pas la même liberté qu'auparavant. Enfin en $\mathbf{1 6 7 0}$ je ne voyais aucun moyen de construire cette église, car nous avions fait bâtir la grande maison où il n'avait plus à faire alors que le dedans, outre le logis où nous étions logés, la grange et autre chose qui avaient beaucoup coûté. Mais dans les peines que j'éprouvai, je promis à la Sainte Vierge de faire bâtir sa chapelle et tout aussitôt je trouvai du soulagement. ${ }^{107}$

Les souffrances intérieures, la nuit de l'esprit commençante, devenait donc dès 1670 , croyons-nous, la rançon dont cette âme d'élite

107. EA., cité dans Faillon, Vie de la Sœur Bourgeoys, 1:204-205. 
allait payer pendant les quelque trente dernières années de sa vie (1670-1700), son ascension dans les voies de la vie unitive. C'est cette pauvre femme qui à l'automne de 1670 se prépare à entreprendre son second voyage en France (1670-1672). Pénible, le voyage le serait beaucoup. Mais, somme toute, la chuse importait peu à l'apôtre, car elle avait pour l'accomplir deux raisons péremptoires: obtenir de Louis XIV des lettres patentes qui donneraient à son Institut la personnalité civile et procéder à un deuxième recrutement de compagnes. ${ }^{108}$ Elle irait donc sa route.

Trente années de vie illuminative (1640-1670), en la plongeant dans la Nuit des sens, avait épuré et affiné sa sensibilité. Depuis la volte-face de 1640, Marguerite avait comme expérimentalement connu le vide des choses créées. L'exercice des vertus et des dons - ceux de prudence, de force, de conseil et de science surtout - l'avaient soutenue tout le long de cette douloureuse époque de la vie spirituelle et ce premier travail d'épuration l'avait portée au vif désir de Dieu qu'elle gémissait de ne pas mieux servir.

Toutefois elle n'était pas encore au terme de son itinéraire; il lui faudrait encore franchir la plus rude étape - les ténèbres de l'esprit celle-là même dont Saint-Jean de la Croix a formulé, d'expérience, le jugement qui suit:

La première purification ou Nuit est amère et redoutable pour le sens... La seconde ne peut lui être comparế; elle n'est qu'horreur et épouvante pour l'esprit. ${ }^{109}$

Yvon Charron, p.s.s.

108. EA., cité dans Faillon, Vie de la Sœur Bourgeoys, 1:212.

109. Voir note 42. 